\title{
THE ORTHOGONAL ELECTROCARDIOGRAM IN LEFT VENTRICULAR HYPERTROPHY
}

\author{
BY \\ E. J. FISCHMANN \\ From the Cardiology Department, Green Lane Hospital, Auckland, New Zealand \\ Received March 26, 1957
}

It has been shown experimentally that three bipolar electrocardiograms from the main axes of the body contain as much or more information about QRS forces than 12-lead electrocardiograms (Frank et al., 1955; Schmitt and Simonson, 1955; and Newman et al., 1955). A clinical comparison of such a three-lead system and the 12 conventional leads has not yet been reported, and one aim of the present study is to make this comparison in left ventricular hypertrophy (LVH).

The three leads under discussion have been used mainly in the synthesis of vector loops. It was shown previously (Fischmann and Brown, 1954; Fischmann, 1955) that much of the behaviour of the spatial vector loop is revealed by inspection of these three leads recorded with a conventional electrocardiograph. To demonstrate this in left ventricular hypertrophy is another aim of the present study.

The description of normal leads in the present paper is incomplete, as the boundary between normal and abnormal conditions other than in left ventricular hypertrophy is not drawn. While a two-channel electrocardiograph was used throughout, useful information may be obtained by single-channel recording.

\section{MATERIAL AND Method}

A hundred and nineteen normal subjects and 42 abnormal subjects were studied. The abnormal group consisted of 4 patients with aortic regurgitation and radiological evidence of cardiac enlargement, and 38 patients in whom repeated estimations over several months have shown that the diastolic pressure was $100 \mathrm{~mm} . / \mathrm{Hg}$ or more. Selection was not affected by radiological or cardiographic evidence of LVH in hypertension or cardiographic evidence of LVH in aortic regurgitation. None of the subjects studied had evidence of ischæmic heart disease, none were under drug therapy, and none showed branch block or an " $X$ " wave in either lead V1 or V2. All were ambulatory and over 20 years of age.

A frontal plane lead-pair (vertical and transverse lead of the Duchosal-Grishman " cube "), and a horizontal plane lead-pair (sagittal and transverse lead) were recorded with a two-channel electrocardiograph (Fischmann and Brown, 1954). The transverse lead was the lower lead of each lead-pair, and served also as a common time base. The lead connections of Shillingford and Brigden (1951) were used; positive deflections in the vertical lead correspond to upward, in the transverse lead to leftward, in the sagittal lead to forward directed cardiac forces. To facilitate visualization the direction is marked in the illustrations, and the lead notation previously used (Fischmann and Brown, 1954) has been replaced by the letters $v$ (vertical), $t$ (transverse), and $s$ (sagittal). These letters are also used in the text for the identification of the deflections (e.g. $\mathrm{R} t$ means the $\mathrm{R}$ wave of the transverse lead). In the vertical lead, positive and negative mean above and below the isoelectric line, without reference to the Einthoven standard lead polarity convention. QRS deflections and S-T deviation in the three leads of all subjects were measured using magnification where necessary and tabulated on a master chart, together with a qualitative description (positive, negative, diphasic, isoelectric) of the T wave. Amplitudes measured in mm., were divided by the amplitude of the calibration excursion (approximately $1 \mathrm{mV}=1.5 \mathrm{~cm}$.) giving voltage in $\mathrm{mV}$. From the 
master chart minima, maxima, and means of some QRS wave, and composite voltages and wave ratios were then determined, and QRS configurations noted. Finally the LVH criteria of Sokolow and Lyon (1949) and Goldberger (1949) were applied to the 12-lead electrocardiograms of the abnormal group.

\section{RESULTS}

Vertical Lead. Three forms of QRS were encountered in this lead. (1) Mainly negative QRS $\left(\mathrm{rS}, \mathrm{rSr} r^{\prime}, \mathrm{Qr}\right)$ frequently found both normally and in LVH (Fig. 1). An abnormal variant could not

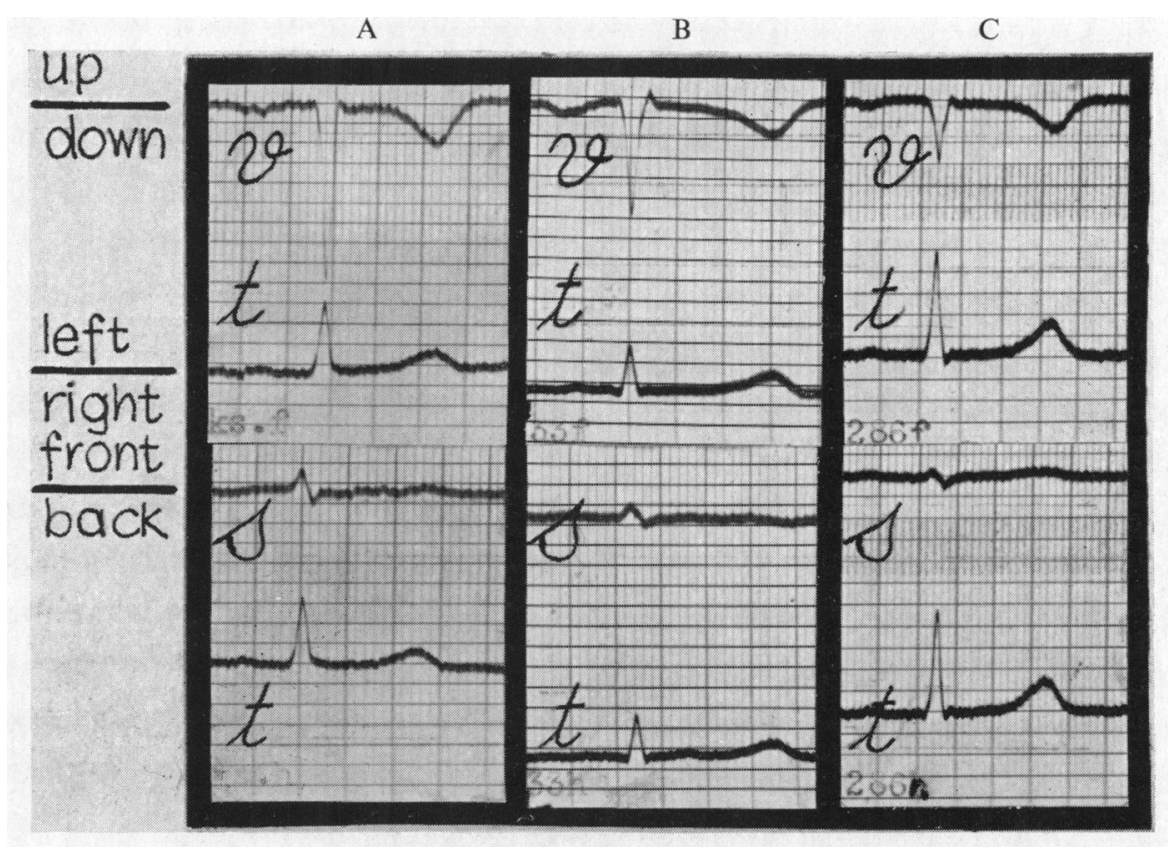

FIG. 1.-Vertical lead QRS mainly negative, transverse lead QRS mainly positive, sagittal lead $Q R S$ symmetrical diphasic (RS), as found in the majority of normal subjects and in some with LVH. (See Table I for incidence). Three normal records. v, vertical; $t$, transverse; $s$, sagittal lead. Thus $\mathrm{R} v=\mathrm{R}$ wave of vertical lead. Vectors: Cardiac depolarization forces, which correspond to the main QRS deflection, are down (lead $v$ ) and left (lead $t$ ) directed.

be defined. (2) Mainly positive QRS ( $q R$ ), rare in normals and common in the abnormal; with $R$ wave in excess of $0.4 \mathrm{mV}$ and $\mathrm{R} / \mathrm{Q}$ ratio of 3 or more found only in the abnormal group (Fig. 2). (3) Bizarre QRS seen in the abnormal group alone (Fig. 5). S-T elevation of 0.05 mV and S-T depression in excess of this were seen in both groups, S-T elevation of over $0.05 \mathrm{mV}$ in abnormals alone. All normal subjects except three in whom QRS voltage was less than $0.5 \mathrm{mV}$, showed negative $T$ waves. Isoelectric $T$ in the presence of $Q R S$ voltage greater than $0.5 \mathrm{mV}$, and diphasic $T$, were found in abnormal subjects alone. The voltage of the $\mathrm{R}$ wave in $\mathrm{LVH}$ at times exceeded the normal maximum $\mathrm{R} v$ voltage.

Transverse Lead. QRS configuration was remarkably constant in this lead, $\mathrm{R}$ being the greatest deflection in all normal and abnormal records. The S-T segment was isoelectric, or elevated by $0.05 \mathrm{mV}$ or less, $\mathrm{T}$ always upright, in normals. $\mathrm{S}-\mathrm{T}$ depression greater than $0.05 \mathrm{mV}$ and negative, isoelectric or diphasic T occurred only in the abnormal. A comparison of the wave voltages of the QRS complex showed no useful difference between normal and abnormal.

Sagittal Lead. Four forms of QRS were encountered in this lead. (1) Symmetrically diphasic QRS with initial positive and terminal negative deflection (RS), common in both normals and the abnormal group (Fig. 3). An abnormal variant could not be defined. (2) Diphasic QRS with 
A

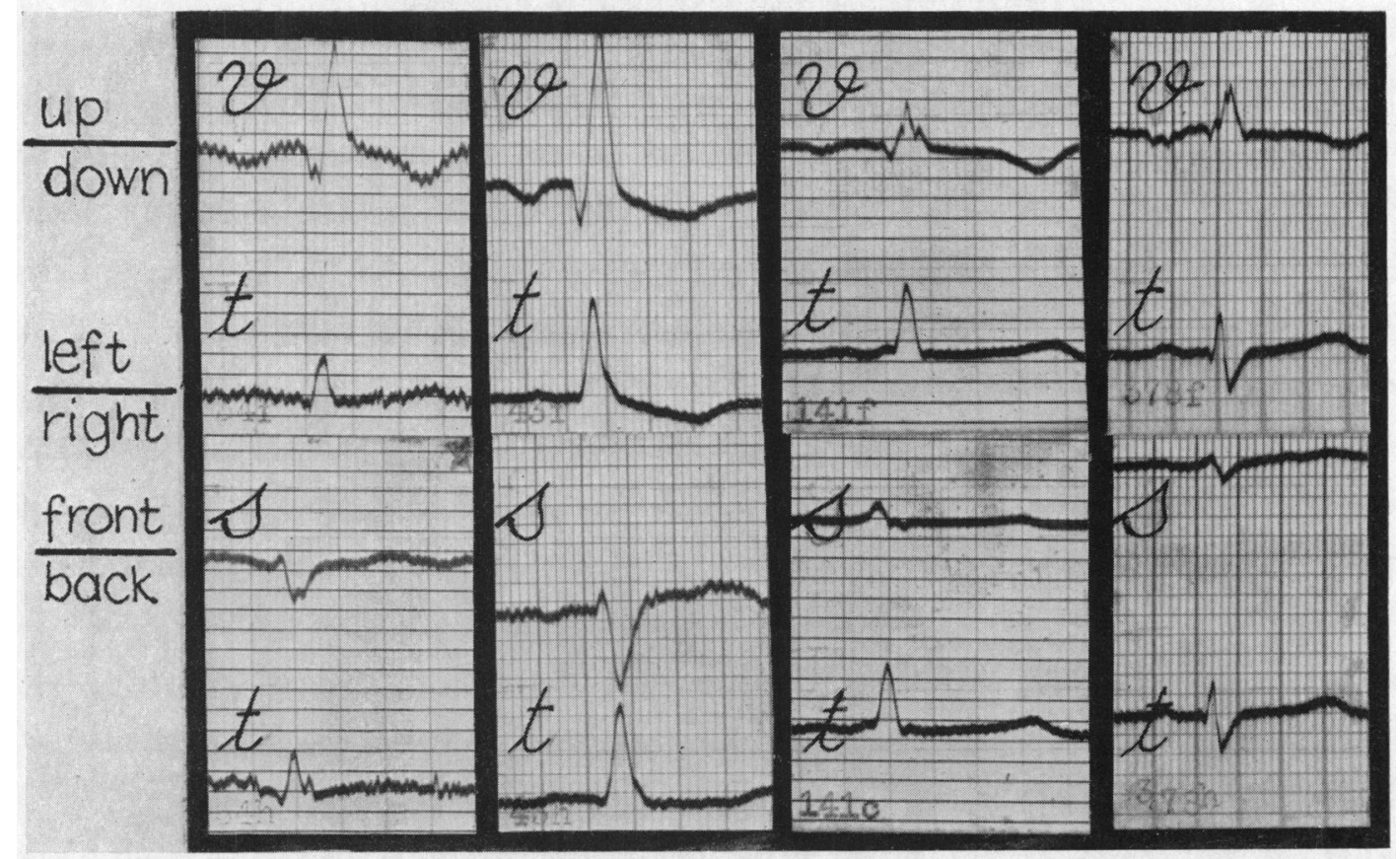

FIG. 2.-Vertical lead QRS mainly positive, as seen in 7 of 119 normals and in 16 of 42 subjects with LVH. Records of 4 abnormal subjects are shown. In LVH Rv exceeds $0.4 \mathrm{mV}$ or vertical lead $\mathrm{R} / \mathrm{Q}$ ratio of 3 is exceeded. Transverse lead S-T and T abnormal in B. Vectors: In record C depolarization forces are up, left and backward directed, almost through the whole of QRS, in the other records shown they are so directed only in the latter part of QRS.

prominent $\mathrm{R}(\mathrm{Rs})$, rare in both normals and the abnormal. An abnormal variant was also not distinguishable. (3) Diphasic QRS with prominent $\mathrm{S}$ (rS), common in the abnormal and less common in normal; with an $\mathrm{S}$ wave in excess of $0.45 \mathrm{mV}$ and an $\mathrm{S} / \mathrm{R}$ ratio in excess of 6 only in the abnormal group (Fig. 3). (4) A QS pattern (Fig. 5B) was seen in two subjects of the abnormal group. Of the waves of the QRS complex $S$ alone showed a useful difference between the normal and abnormal voltage range. In view of the relatively low voltage and the unsteady baseline in this lead the $\mathrm{S}-\mathrm{T}$ segment was of no diagnostic value. Positive and negative $\mathrm{T}$ waves occurred in both normal and abnormal subjects.

Composite voltage measurements were calculated and $\mathrm{R} v+\mathrm{R} t$ and $\mathrm{R} t+\mathrm{S} s$ were significant; the former showed an upper normal limit of $1.3 \mathrm{mV}$ was exceeded in 5 cases, and the latter showed an upper normal limit of $1.5 \mathrm{mV}$ which was exceeded in two abnormal subjects: all of these 7 subjects showed other abnormalities in one or more of the three leads.

Vectorcardiographic data obtained by inspection and correlation of the three leads. This attempts to show, without assessing the incidence and validity of diagnostic criteria, that the behaviour of QRS and T vectors and vector loops in LVH is evident from the 3 leads. The spatial QRS vector loop in LVH (Duchosal and Sulzer, 1949; Scherlis et al., 1951; Gardiner and Lowe, 1953; Horan et al., 1954; Portheine, 1955; Wenger, 1955) is left, up, and backward orientated; its frontal projection often wide and inscribed counterclockwise; in the horizontal plane the loop may be wide and is normally inscribed. Frequently only the later portions of the loop are upward (Gardiner and Lowe, 1953). Wolff et al. (1953) found counterclockwise rotation round the long axis of the heart the most constant change in LVH. This resulted in an exaggerated disposition of initial forces right and forward and with reduced upward direction. The T loop in LVH deviates from the QRS loop and there may be a shift of the junction $J$. 


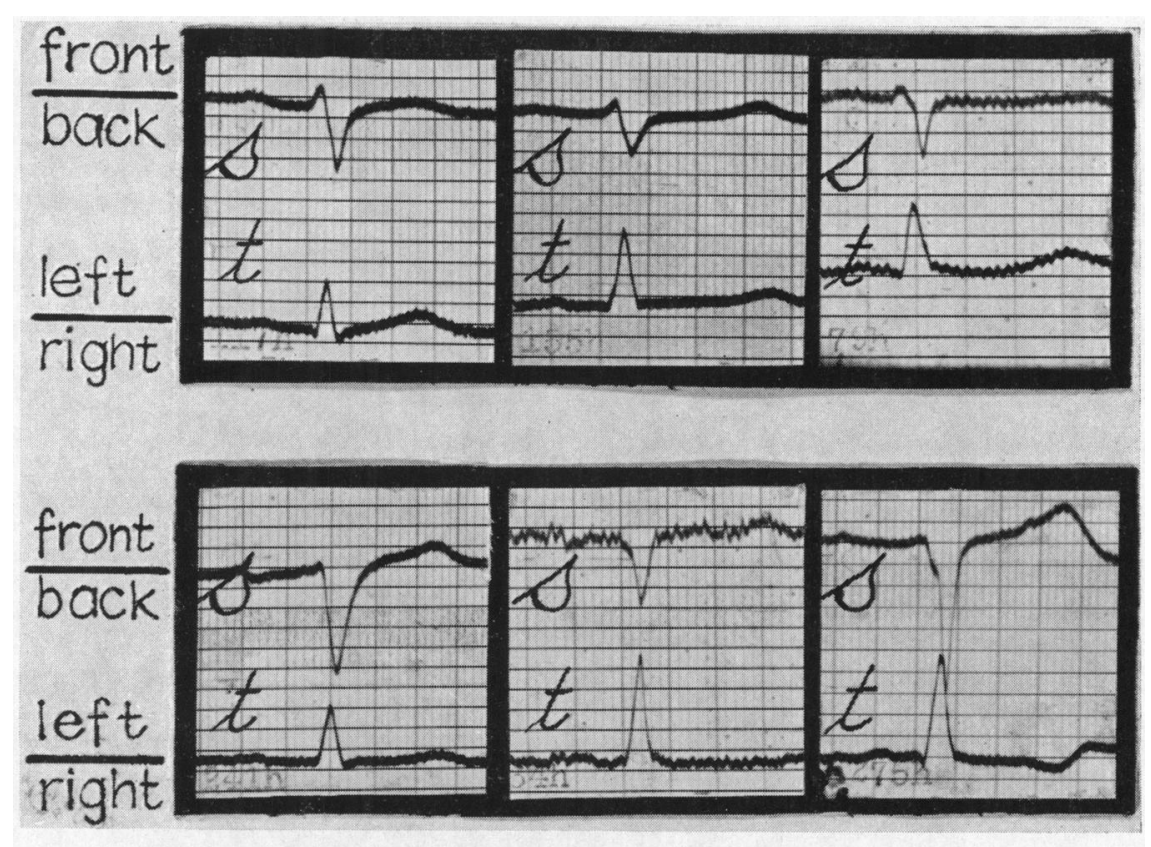

FIG. 3.-Sagittal lead QRS mainly negative, as seen in 20 of 119 normal subjects and in 28 of 42 with LVH. Horizontal plane $(s, t)$ lead-pairs of three normal subjects (first row) and of three with LVH (second row) are shown. Abbreviations as Fig. 1 . In the sagittal lead the $S$ wave exceeds $0.45 \mathrm{mV}$ (Calibration approximately $1.5 \mathrm{~cm} . / \mathrm{mV}$ ) and the $S / R$ ratio exceeds 6 , in abnormal subjects only. Transverse lead $S-T$ and $T$ are abnormal in the last two records.

Fig. 4 shows lead-pair records in up, left and backward orientation of the QRS loop. In the first two records of the figure, the lateness of the $\mathrm{R} v$ and $\mathrm{Q} s$ peaks shows that up and backward displacement affected mainly the latter parts of the loop, whereas asynchronism of the main QRS peaks in these records shows increased width of both the frontal and horizontal plane loops. This may be compared with the normal records in Fig. 1, where near-synchronous or synchronous peaks indicate narrow QRS loops (Fischmann and Brown, 1954). In all frontal plane $(v, t)$ lead-pairs of Fig. 2 and 4 the peak of $\mathrm{R} t$ (left), precedes the $\mathrm{R} v$ (upward) peak. This means that the direction of inscription in at least the distal part of the QRS loops was first left then upward, i.e. counterclockwise. In all records of the normal and in all but two of the abnormal group, initial QRS vectors were forward directed, shown by the initially positive sagittal lead QRS. In addition the initial vectors were directed to the right, giving rise to a small q wave in the transverse lead (Fig. 2A; Fig. 4), or leftward, causing transverse lead QRS to commence with the upstroke of R (Fig. 1A and 5B). Upward orientation of the initial vectors causes a small initial $r$ wave in the vertical lead (Fig. 1B), whereas downward initial vectors correspond to a $q$ or $Q$ wave in this lead (Fig. 2). Divergence of the QRS and $T$ loops causes discordance of the main QRS deflection and the T wave, in one or more of the three leads, abnormal shifts of the junction $\mathrm{J}$ cause the $\mathrm{S}-\mathrm{T}$ segment abnormalities described in the preceding section.

\section{Discussion}

Of the 42 abnormal subjects 37 showed the signs of left ventricular hypertrophy described by Sokolow and Lyon (1949) and 33 showed the criteria described by Goldberger (1949). In the three leads of the " cube" system, 34 abnormal subjects presented changes not encountered in the normal (Table I). 31 of the 34 subjects showing changes in the "cube" leads also showed LVH in the 


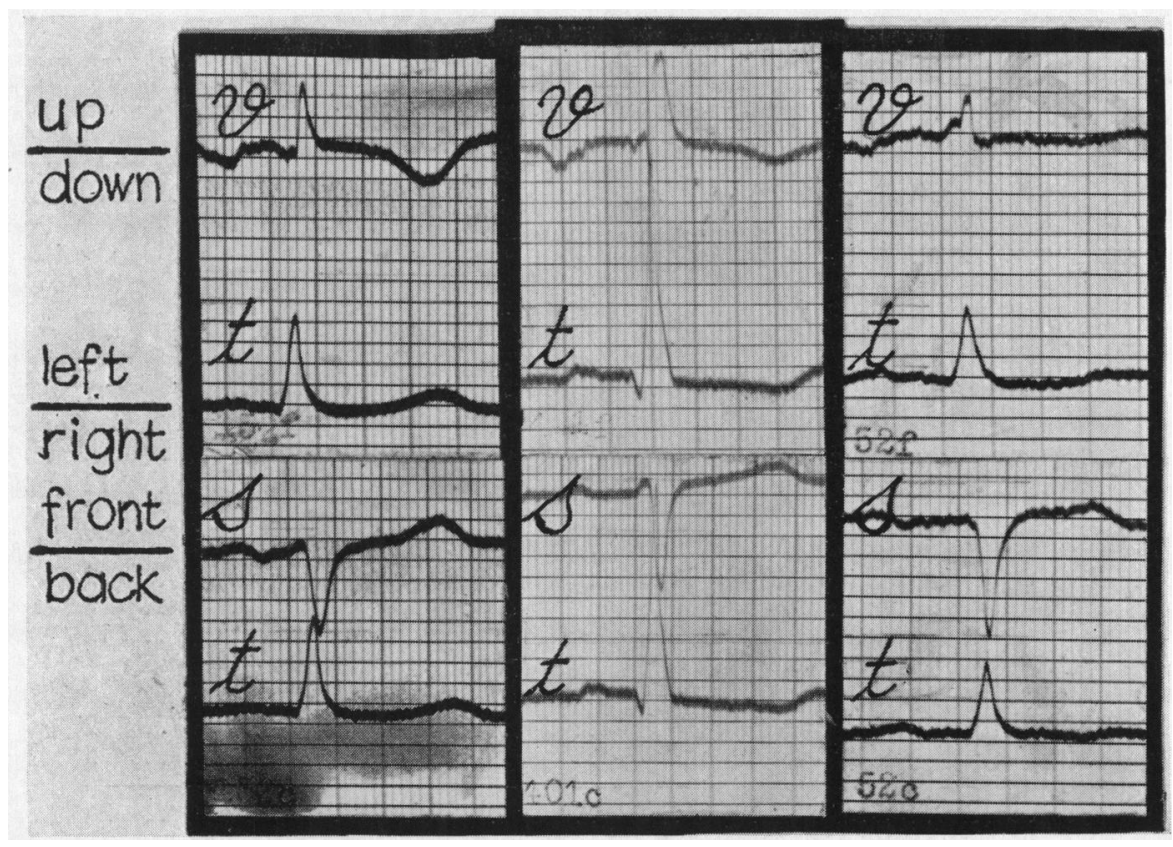

FIG. 4.- $Q R S$ in vertical lead mainly positive, in sagittal lead mainly negative. 3 records from subjects with left ventricular hypertrophy, combining the QRS anomalies shown in the two preceding Figs. Abbreviations as Fig. 1. Calibration approximately $1.5 \mathrm{~cm} . / \mathrm{mV}$. Abnormal transverse lead $\mathrm{S}-\mathrm{T}$ depression with $\mathrm{T}$ inversion in the second and with abnormal isoelectric $\mathrm{T}$ in the third record. Vectors: Depolarization forces corresponding to the main QRS deflection are up, left and backward directed. Initial forces in the two first records are down, right and forward, in the last record up, right and backward. A wide horizontal $(s, t)$ plane QRS loop is indicated by asynchronism of the sagittal and transverse lead main QRS peaks. The frontal $(v, t)$ plane vector loop is counter-clockwise inscribed in all records, as $\mathrm{R} t$ (leftward) precedes $\mathrm{R} v$ (upward). The orientation of the $\mathrm{T}$ wave in the last two records is abnormal: right and forward.

12-lead electrocardiogram. In spite of the disadvantages of the transverse lead of the "cube" system, the incidence of $\mathrm{LVH}$ changes in the three leads is comparable with the incidence in the conventional cardiogram. In addition the three leads supply information concerning the vectorcardiogram.

Apart from the initial and at times the terminal phases of ventricular depolarization, QRS forces are normally left and downward directed. In left ventricular hypertrophy, increase of the muscle mass and rotation of the heart around its longitudinal and anteroposterior axes, may displace these forces up and backward. If left ventricular activation is delayed by impairment of intraventricular conduction, left ventricular forces are unopposed by the normally partly synchronous, down and forward directed, right ventricular forces. This again will facilitate up and backward deviation of QRS forces. As a result of cardiac dipole eccentricity, horizontal QRS forces may appear upward directed (Gardberg, 1954). Finally, using the "cube" lead system the vertical component of the cardiac force is augmented whilst the transverse and sagittal components are foreshortened (Frank and Kay, 1955). This results in augmentation of upward directed cardiac forces.

In the vertical lead, normal downward orientation of QRS forces causes a predominantly negative QRS complex (Fig. 1). When QRS forces are upward directed the vertical lead becomes predominantly positive. This happens occasionally in controls and, to a greater degree (as shown by the abnormal $R / Q$ ratio and $R$ voltages) in subjects with left ventricular hypertrophy (Fig. 2). Owing to leftward QRS force orientation in both normal and in the abnormal group, the 
TABLE I

Findings not encountered in 119 normal subjects, and the incidence of these findings in 42 subjects with left ventricular hypertrophy. $\mathrm{R} t$, transverse lead $\mathrm{R}$ wave; $\mathrm{S} s$, sagittal lead $\mathrm{S}$ wave; $\mathrm{R} v$, vertical lead $\mathrm{R}$ wave.

Vertical lead:

$\mathrm{R}$ wave greater than $0.4 \mathrm{mV}$.

$\mathrm{R} / \mathrm{Q}$ ratio 3 or more ..

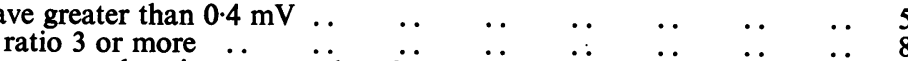

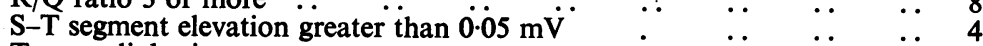

T wave diphasic

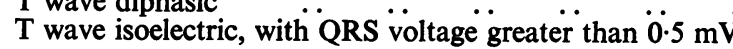

Transverse lead:

S-T segment depression by $0.05 \mathrm{mV}$ or more

T wave diphasic

T wave isoelectric

$\mathrm{T}$ wave negative

Sagittal lead:

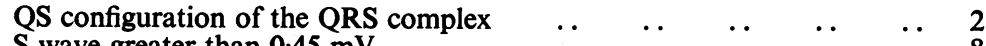

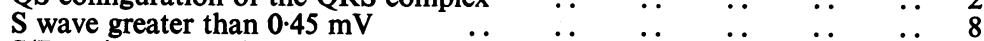

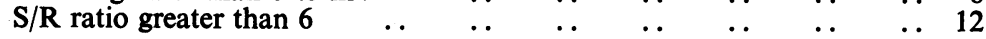

Composite voltages:

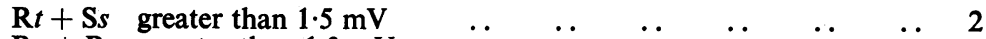

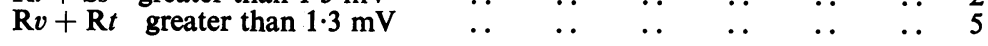

$\begin{array}{llllllll}\text { Total showing ST,T changes } . . & \ldots & \ldots & \ldots & \ldots & \ldots & \ldots & 26\end{array}$

$\begin{array}{lllllllll}\text { Total showing QRS changes } \ldots & \ldots & \ldots & \ldots & \ldots & \ldots & \ldots & 18\end{array}$

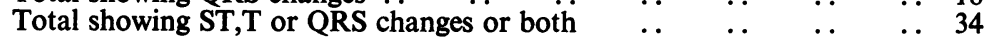

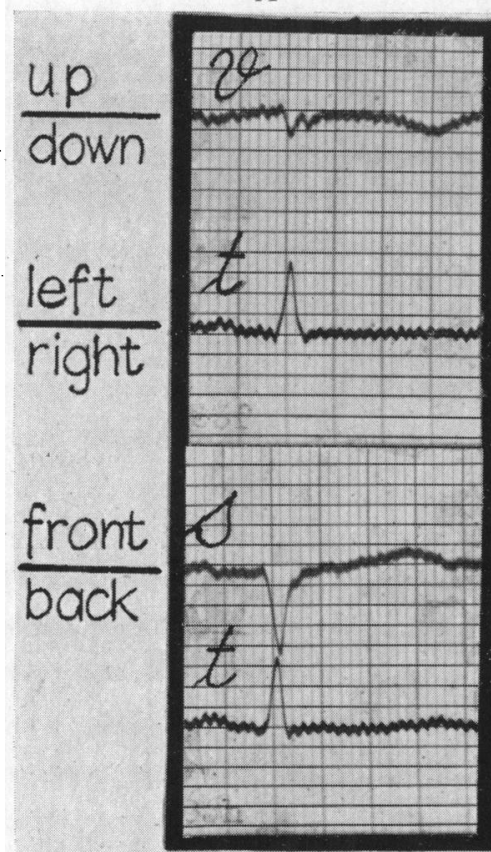

B

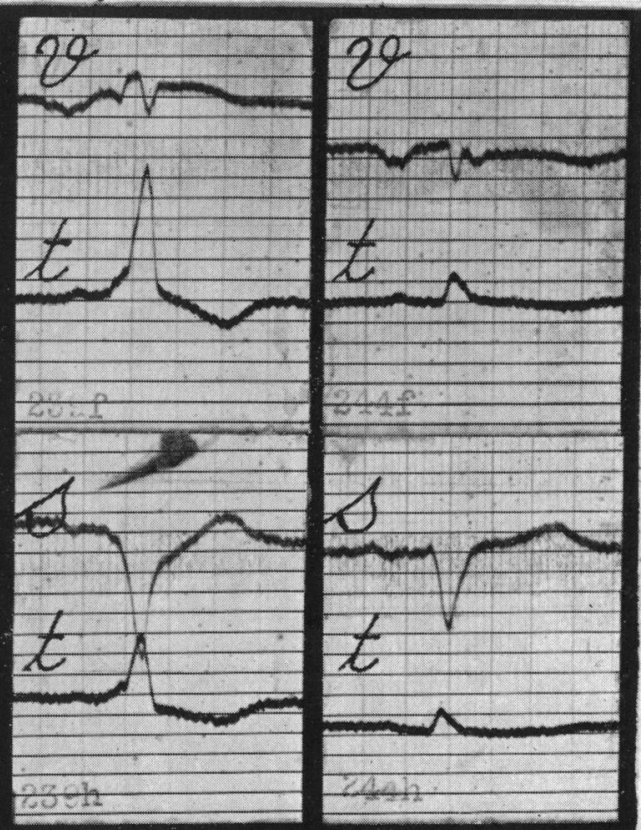

Fig. 5.-Bizarre vertical lead $Q R S$, as seen in 3 subjects of the $\mathrm{LVH}$, and in none of the normal, group. Abnormal isoelectric $T$ wave in the transverse lead of record $A$, abnormal inverted transverse lead $T$ wave in records $B$ and $C$, abnormal elevated vertical lead S-T segment in record B. Abbreviations as in Fig. 1. 
transverse lead displays a prominent $\mathrm{R}$ wave. In the sagittal lead, the backward orientation of forces in LVH causes a mainly negative QRS complex (Fig. 3). Such a QRS complex was also found in some normal subjects, but without the abnormal S/R ratio and S voltages seen in LVH. It is difficult to explain the bizarre QRS complex in the vertical leads of three of the abnormal group (Fig. 5). Incomplete left bundle-branch block is a possibility, particularly in Fig. 5B and C; since in the former QRS duration is $0.11 \mathrm{sec}$. and, as shown by the positive QRS onset, the initial forces are leftward directed in both records.

Of all the calculated quantities within the QRS complex the R/Q ratio in the vertical, and the $S / R$ ratio in the sagittal lead were the most useful. That ratios of opposing forces may prove a useful substitute for lead voltage and vector magnitude measurement, and a temporary short-cut to the solution of the lead problem until more accurate leads than those now in use are found is suggested by the following. The voltage in a lead is, owing to body non-homogeneity and surface configuration and to eccentricity of the cardiac dipole, not a simple geometric projection of the dipole on the lead axis, but a scalar product of that projection and the lead vector of Burger and van Milaan (1948). Since the lead vector varies from lead to lead and is unknown in the living subject, absolute voltages in leads are devoid of quantitative meaning in relation to the cardiac dipole force. On the other hand and there is no evidence to the contrary, if the lead vector were constant throughout QRS, any ratio of two voltages within one QRS complex would contain the same lead vector in both denominator and numerator. The ratio is therefore independent of the lead vector and consequently of the inaccuracies of which this vector is a collective expression.

In left ventricular hypertrophy the $\mathrm{R}$ wave in leads aVL, V5, and V6 may show abnormally high voltage. In the present abnormal group of 42 subjects $R$ in lead aVL was $11 \mathrm{~mm}$. or greater in sixteen instances; RV5 or RV6 $26 \mathrm{~mm}$. or more in four instances (Sokolow and Lyon, 1949); aVL $13 \mathrm{~mm}$. or more (Goldberger, 1949), in twelve instances. As leads aVL, V5, and V6 have approximately transverse lead axes, high voltage of the transverse lead of the "cube" lead system was also expected. In fact the transverse lead of the "cube" failed in this respect for the normal and abnormal voltages of the $\mathrm{R}$ wave in this lead overlap and it was not possible to define a normal maximum value. This observation is in keeping with the fact that past published work on the vectorcardiogram of left ventricular hypertrophy, employing the Duchosal-Grishman " cube " has not yielded a quantitative definition of QRS force magnitude in left ventricular hypertrophy. It is also to be expected from the fact that the axis of the transverse lead of the " cube " deviates clockwise from an ideal transverse lead (Frank, 1955; Schaffer, 1956) whereas the axis of lead aVL (and lead I) deviates counterclockwise. As LVH forces often point left and upward, they tend to parallel the axes of aVL and lead I and to lie perpendicularly to the transverse lead of the " cube." This could result in augmentation of these forces by the former leads and in attenuation by the latter lead. The opposite will apply to normal left and downward directed forces. Thus departing from an ideal transverse lead, leads I and aVL exaggerate, the transverse " cube " lead reduces the quantitative difference between normal and LVH forces. In addition the transverse lead of the "cube" foreshortens transverse cardiac forces (Frank, 1955), thereby acting as a short and relatively insensitive scale for their measurement.

The data on which the diagnosis of LVH was based in the present study, namely QRS configuration, voltage measurement, and S-T, T changes, would all be available had leads been recorded consecutively with a single-channel instrument. On the other hand, with regard to vectorcardiographic data, it was found that if consecutively recorded leads were aligned in a manner suggested by Goldberger (1953), the direction of vectors could be determined with reasonable accuracy but the direction of loop inscription was occasionally reversed by the procedure of alignment.

\section{SUMMARY}

The three leads of the cube lead system and the conventional 12-lead electrocardiogram were recorded with a scalar electrocardiograph in 119 control and in 42 subjects with arterial hypertension or aortic regurgitation. The three leads of the cube were so connected that positive 
deflections in the vertical lead meant upward, in the transverse lead leftward, and in the sagittal lead forward, directed cardiac forces.

The QRS complex of the vertical lead was negative ( $\left.\mathrm{rS}, \mathrm{rSr}^{\prime}, \mathrm{Qr}\right)$, owing to downward $\mathrm{QRS}$ forces, in 112 normal and 21 abnormal subjects. It was positive (qR), owing to upward directed forces, in 7 normal and in 16 abnormal subjects but $q R$ configuration with a R/Q ratio of 3 or more and an $\mathrm{R}$ wave greater than $0.4 \mathrm{mV}$ occurred only in the abnormal group.

Owing to leftward directed ventricular forces the QRS complex of the transverse lead was positive (qR, qRs, Rs) in all normal and abnormal records.

The QRS complex of the sagittal lead showed Rs or RS configuration in 99 normal and 14 abnormal subjects. Owing to backward directed forces, it was mainly negative (rS) in 20 normal and in 28 abnormal subjects but $\mathrm{rS}$ configuration with an $\mathrm{S} / \mathrm{R}$ ratio greater than 6 and an $\mathrm{S}$ wave greater than $0.45 \mathrm{mV}$ occurred only in the abnormal group. QS configuration in the sagittal lead, caused by totally backward QRS forces, was found only in two abnormal subjects.

$\mathrm{R} v+\mathrm{R} t$ exceeded $1.3 \mathrm{mV}$ in five, and $\mathrm{R} t+\mathrm{S} s$ exceeded $1.5 \mathrm{mV}$ in two subjects of the abnormal and in none of the normal group.

The QRS pattern and voltage changes enumerated, together with S-T/T changes described in the text, allowed the diagnosis of left ventricular hypertrophy in 34 of the 42 subjects in the abnormal group. The 12-lead electrocardiogram permitted this diagnosis in 33 to 37 subjects depending on the criteria for diagnosis of LVH which were used.

Conventional leads with near-transverse lead axes (lead I, aVL), distinguish between the normal and left ventricular hypertrophy in terms of QRS voltage. The transverse lead of the cube fails to do so.

The $R / Q$ ratio in the vertical and the $S / R$ ratio in the sagittal lead are more useful in the diagnosis of $\mathrm{LVH}$ from three orthogonal leads, than absolute QRS voltage measurements. Reasons are given for the view that ratios of forces within a single QRS complex are more independent of the error introduced by body characteristics and by dipole eccentricity than are absolute voltages.

The essential vectorcardiographic criteria of left ventricular hypertrophy may be recognized by inspection of the three leads.

It is a pleasure to acknowledge the valuable technical assistance of Mr. A. Fischman and Miss Marlene Watson.

\section{REFERENCES}

Burger, H. C., and van Milaan, J. B. (1948). Brit. Heart J., 10, 229.

Duchosal, P. W., and Sulzer, R. (1949). La Vectorcardiographie. Bale.

Fischmann, E. J., and Brown, D. (1954). Brit. Heart J., 16, 351.

- E. J. (1955). Brit. Heart J., 17, 496.

Frank, E., Kay, C. F., Seiden, G. E., and Keisman, R. A. (1955). Circulation, 12, 406.

- and Kay, C. F. (1955). Amer. Heart J., 49, 670.

Gardberg, M. (1954). Circulation, 10, 544.

Gardiner, J. M., and Lowe, T. E. (1953). Australasian Ann. Med., 2, 22.

Goldberger, E. (1949). Unipolar Lead Electrocardiography. 2nd ed., Philadelphia. (1953). Unipolar Lead Electrocardiography and Vectorcardiography. Philadelphia.

Horan, L. G., Burch, G. E., Abildskov, J. A., and Cronvich, J. A. (1954). Circulation, 10, 728.

Newman, V. E., McGovern, J. F., and Arnold, T. G. (1955). Arch. intern. Med., 96, 591.

Portheine, H. (1955). Zeitschr. Kreislaufforschg., 44, 368.

Schaffer, A. I. (1956). Amer. Heart J., 51, 588.

Scherlis, L., Grishman, A., Sandberg, A. A., and Dvorkin, J. (1951). Amer. Heart J., 41, 494.

Schmitt, O. K., and Simonson, E. (1955). Arch. intern. Med., 96, 574.

Shillingford, J., and Brigden, W. (1951). Brit. Heart J., 13, 233.

Sokolow, M., and Lyon, T. P. (1949). Amer. Heart J., 37, 161.

Wenger, R. (1956). Klinische Vektorkardiographie. Darmstadt.

Wolff, L. (1955). Dis. Chest, 27, 263.

-, Richman, J. L., and Soffe, A. M. (1953). New England J. Med., 246, 810 and 851. 